\section{Buchrezension zu: Die geheimnisvolle Macht der Farben}

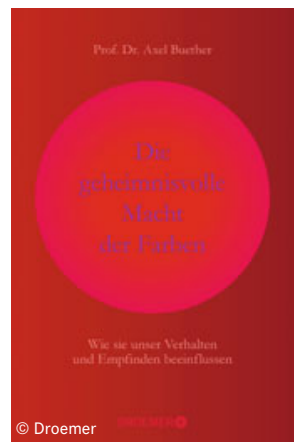

Die geheimnisvolle Macht der Farben

Wie sie unser Verhalten und Empfinden beeinflussen

Axel Buether

320 S., Droemer, 2020.

$H C, 25,-€$

ISBN: 9783426277874

Auch als E-Book erhältlich

DOI: $10.1007 / \mathrm{s} 12268-021-1582-8$

(C) Der Autor 2021

Warum fühlen wir uns in Räumen wohl? Warum wirken Farben hier passend und dort nicht? Farben sind für viele Menschen eine Geschmacksfrage - gefällt oder gefällt nicht. Das Warum dahinter wird oft kaum reflektiert und hinterfragt. Die Mechanismen der Farbwirkung sind häufig unbewusst und schwer mit offensichtlichen Wenn-Dann-Beziehungen zu erklären. Die Wirkung der Farbe wird daher oft mit einer geheimnisvollen Macht verglichen, die einen Zauber beinhaltet, den wir kaum erklären können.

Für Prof. Dr. Axel Buether versteckt sich hinter der Farbe in unserer Umwelt eine deutliche Zeichenebene, die als ein Kommunikationssystem verstanden werden kann, das den Lebewesen auf der Erde hilft, uns in der Welt und der Gemeinschaft zurecht zu finden und Orientierung zu erfahren. Tatsächlich geht die Wirkung der Farben weit über die persönliche Beurteilung eines jeden einzelnen Menschen hinaus. Die Tatsache, dass Farbwirkungen zu einem großen Teil auf vergleichbaren biologischen und sinnlichästhetischen Wahrnehmungen beruhen und zum Teil sozialisiert werden, ist ein Indiz für die Notwendigkeit der ganzheitlichen Betrachtung der Farbwirkung mit Schnittstellen in fast alle anderen Wissenschaften wie zum Beispiel Design und Architektur. Farbe ist nicht nur meine persönliche Wahrnehmung, sondern ein aktives und veränderliches, wenn auch verlässliches, Kommunikationssystem innerhalb von Gruppen und ganzen Gesellschaften. Die Reflexion über das Phänomen Farbe kann folglich auch helfen, bewusst über die eigene Wahrnehmung, das eigene Handeln und die eigene Position in der Welt und ihrer Zusammenhänge nachzudenken.

Das Buch von Axel Buether veranschaulicht besonders die biologische, sinnliche und gesundheitliche Ebene der Farbe auf eine informative, fundierte und gleichzeitig mitreißende Art. Vielfältige wissenschaftliche Erkenntnisse inklusive ihrer Quellen begleiten die plastisch nachvollziehbaren und klar geschriebenen Texte. Sehr zu empfehlen ist das Buch allen, die nicht nur über die Wirkung von Farbe mehr wissen wollen, sondern allen Leserinnen und Lesern, welche die Komplexität unserer Wahrnehmung und unseres Verhaltens besser verstehen und in bewusste Handlungen zur Gestaltung ihrer Umwelt übersetzen möchten. Ein exzellentes Buch, das die geheimnisvolle Macht der Farben nachvollziehbar macht und verständlich erklärt.

Timo Rieke,

HAWK, Fakultät Gestaltung, Hildesheim, timo.rieke@hawk.de

Diese Rezension erscheint Open Access. *
Funding note: Open Access funding enabled and organized by Projekt DEAL. Open Access: Dieser Artikel wird unter der Creative Commons Namensnennung 4.0 International Lizenz veröffentlicht, welche die Nutzung, Vervielfältigung, Bearbeitung, Verbreitung und Wiedergabe in jeglichem Medium und Format erlaubt, sofern Sie den/die ursprünglichen Autor(en) und die Quelle ordnungsgemäß nennen, einen Link zur Creative Commons Lizenz beifügen und angeben, ob Änderungen vorgenommen wurden. Die in diesem Artikel enthaltenen Bilder und sonstiges Drittmaterial unterliegen ebenfalls der genannten Creative Commons Lizenz, sofern sich aus der Abbildungslegende nichts anderes ergibt. Sofern das betreffende Material nicht unter der genannten Creative Commons Lizenz steht und die betreffende Handlung nicht nach gesetzlichen Vorschriften erlaubt ist, ist für die oben aufgeführten Weiterverwendungen des Materials die Einwilligung des jeweiligen Rechteinhabers einzuholen. Weitere Details zur Lizenz entnehmen Sie bitte der Lizenzinformation auf http://creativecommons.org/licenses/ by $/ 4.0 /$ deed.de. 